\title{
Properties of junction diodes under conditions of bisotropic strains
}

\author{
V.L. Borblik \\ V. Lashkaryov Institute of Semiconductor Physics, National Academy of Sciences of Ukraine, \\ 41, prospect Nauky, 03028 Kyiv, Ukraine \\ Phone: +38(044)525-6292, fax: +38(044)525-7463
}

\begin{abstract}
In consideration of influence of technological strains on characteristics of junction diodes located on surface of silicon wafers, biaxial character of such strains has taken into account. The cases of (001)- and (111)-oriented silicon wafers on whose surface the diodes are located as well as longitudinal (in plane of the wafer) and transversal (perpendicular to it) diode current flow have been considered. It is shown that at small strains, the diodes located on the surface of (111)-Si are less subjected (as a whole) to their influence. Furthermore, the change in the intrinsic carrier concentration has been assessed in (001)-Si at very large bisotropic strains. The concentration is shown to increase by several orders of magnitude irrespective of the strain sign.
\end{abstract}

Keywords: junction diode, bisotropic strain, piezojunction effect, silicon, SiGe alloy.

Manuscript received 15.12.08; accepted for publication 18.12.08; published online 30.01.09.

\section{Introduction}

In the papers [1-6], an influence of mechanical stresses on characteristics of $p-n$ diodes and transistors (piezojunction effect) was studied. In a number of cases, motivation of these investigations was to study effect of technological deformations on performance of $p$ - $n$ diodes (or transistors) used as temperature sensors. Herewith the mechanical stresses were considered as uniaxial (sometimes as hydrostatic).

In the same time, it is clear that in multi-layer structures (diodes, transistors) which consist of a substrate, doped layers, passivating and metallic coatings, etc., biaxial deformations develop acting in plane of the layers. Of course, in response to this action, the uniaxial strain (as a rule, of opposite sign) appears in direction perpendicular to the layers, but initial (given) deformation is still biaxial. Character of the initial strain manifests itself quantitatively and even sometimes qualitatively (see, for example, [7]). Let us notice also that there are mechanical systems which develop controllable external biaxial stresses [8-10].

Only one paper [11] is known to the author, which proceeds from biaxial character of the strain acting to a $p$ - $n$ junction. But in the paper [11], a case of silicon oriented only in [001] direction has been considered and electron transport was assumed in the same direction.
In this paper, an influence of small biaxial isotropic (bisotropic [12]) strains on properties of $p-n$ diodes located on surface of silicon wafer both (001)- and (111)-oriented has been studied theoretically. The effect depends essentially on the wafer orientation and current direction. Furthermore, for (001)-oriented silicon the case of very large bisotropic strains has been considered.

\section{Starting relations}

As it has been shown by us in papers $[4,5]$ effect of deformation on magnitude of a voltage drop across a diode, through which (under its use as temperature sensor) a constant stabilized current is conducted, is analyzed conveniently by means of a product

$$
\frac{n_{\text {min or }}(\hat{u})}{n_{\text {min or }}(0)} \frac{\mu_{\text {min or }}(\hat{u})}{\mu_{\text {min or }}(0)}=\frac{n_{i}^{2}(\hat{u})}{n_{i}^{2}(0)} \frac{\mu_{\text {min or }}(\hat{u})}{\mu_{\text {min or }}(0)}
$$

where $n_{\text {minor }}(\hat{u})$ and $\mu_{\text {minor }}(\hat{u})$ are concentration and mobility of minority carriers in the diode base depending on the strain tensor $\hat{u}, n_{i}(\hat{u})$ is intrinsic carrier concentration in diode material.

When both ratios in (1) vary with strain in the same direction (i.e. together increase or together decrease), the effect is particularly large. But if they vary in opposite manner, then two components of piezojunction effect 
(i.e. changes in concentration and mobility) compensate each other to a high degree. Variation in the voltage drop $\Delta U$ across the diode at the expense of the strain is expressed in the terms of (1) in the form

$$
\Delta U(\hat{u})=-\frac{k T}{q} \ln \left[\frac{n_{i}^{2}(\hat{u})}{n_{i}^{2}(0)} \frac{\mu_{\min \text { or }}(\hat{u})}{\mu_{\min \text { or }}(0)}\right],
$$

where $k$ is the Boltzmann constant, $T$ is temperature, $q$ is the elementary charge. Here and below, we consider only $n^{+}-p$ silicon diode with a short base (when the base thickness is noticeably less than the diffusion length of the minority electrons).

\section{The case of (001)-oriented silicon wafer}

In this case $u_{x x}=u_{y y}=u_{I I}, u_{z z}=u_{\perp}=\frac{2 S_{12}}{S_{11}+S_{12}} u_{I I}$

( $S_{i j}$ are the components of the elastic compliance matrix), and all off-diagonal elements of the strain tensor are equal to zero. Herewith 3 pairs of electron valleys located along [100], [010], and [001] axes acquire following shifts in the energy

$$
\begin{aligned}
& \delta E_{c}^{(100)}=\delta E_{c}^{(010)}=\Xi_{d}\left(2 u_{\|}+u_{\perp}\right)+\Xi_{u} u_{\|}, \\
& \delta E_{c}^{(001)}=\Xi_{d}\left(2 u_{\|}+u_{\perp}\right)+\Xi_{u} u_{\perp},
\end{aligned}
$$

where $\Xi_{d}$ and $\Xi_{u}$ are deformation potentials for the conduction band.

For the shifts in the energy of different branches of the valence band at wave vector $\vec{k}=0$ ( $h$ for heavy holes, $l$ for light holes, $s$ - $o$ for split-off holes), we have (in accordance with $[13,14]$ )

$$
\begin{aligned}
& \delta E_{v}^{h}=a\left(2 u_{\|}+u_{\perp}\right)+\varepsilon, \\
& \delta E_{v}^{l}=a\left(2 u_{\|}+u_{\perp}\right)- \\
& -\left(\varepsilon+\Delta_{s-o}-\sqrt{9 \varepsilon^{2}+\Delta_{s-o}^{2}-2 \varepsilon \Delta_{s-o}}\right) / 2, \\
& \delta E_{v}^{s-o}=a\left(2 u_{\|}+u_{\perp}\right)- \\
& -\left(\varepsilon+\Delta_{s-o}+\sqrt{9 \varepsilon^{2}+\Delta_{s-o}^{2}-2 \varepsilon \Delta_{s-o}}\right) / 2-\Delta_{s-o},
\end{aligned}
$$

where $\varepsilon=-b\left(u_{\|}-u_{\perp}\right), \Delta_{s-o}$ is the energy of spinorbital splitting of the valence band, $a$ and $b$ are the deformation potentials for the valence band.

By means of the self-consistent procedure of calculating the Fermi-energy shift with deformation described in [2], we obtain where $m_{h}, m_{l}$ and $m_{s-o}$ are density-of-state effective masses for heavy, light and split-off holes, respectively, whose variation with the strain at considered here small deformation is neglected (see [4, 5]).

Mobility of the minority carriers (in the given case, of electrons) depends on direction of the current flow. The change in their mobility (if they are considered like majority carriers) occurs in accordance with the expression

$$
\mu_{n}(\hat{u})=\sum_{m=1}^{3} \frac{n^{(m)}(\hat{u})}{n(0)} \mu_{n}^{(m)},
$$

where $n^{(m)}(\hat{u})$ is population of a pair of the valleys located on the different axes of $\langle 100\rangle$-type, $\mu_{n}^{(m)}$ is the electron mobility along the current direction in each of three pairs of these valleys, $n(0)$ is the total electron concentration. Accordingly, at the current in plane of the wafer (along [100] or [010] axis) we obtain

$\frac{\mu_{n}^{\text {long }}(\hat{u})}{\mu_{n}(0)}=\frac{3}{2 K+1} \frac{1+K+K \exp \left(\beta u_{\|}\right)}{2+\exp \left(\beta u_{\|}\right)}$,

and at the current perpendicular to the wafer surface (i.e. along [001] axis) -

$$
\frac{\mu_{n}^{\text {trans }}(\hat{u})}{\mu_{n}(0)}=\frac{3}{2 K+1} \frac{2 K+\exp \left(\beta u_{\|}\right)}{2+\exp \left(\beta u_{\|}\right)}
$$

where $K=\frac{\mu_{t}}{\mu_{l}} \approx \frac{m_{l}}{m_{t}}$ is anisotropy parameter for electron mobility ( $m_{l}$ and $m_{t}$ are longitudinal and transversal electron effective masses, respectively), $\beta=\frac{\Xi_{u}\left(S_{11}-S_{12}\right)}{k T\left(S_{11}+S_{12}\right)}$.

For numerical calculations, the same values of silicon parameters (as in $[4,5]$ ) are used. In Figs. 1 and 2, two components of the piezojunction effect as functions of the bisotropic strain $u_{\|}$are represented (at room temperature): in Fig. 1 - for squared intrinsic carrier concentration (curve “(001)-wafer”), in Fig. 2 for electron mobility at two current directions: in the plane of the wafer (longitudinal) and perpendicular to it (transversal). ${ }^{1}$ Because the mobility in the transversal direction varies with the strain qualitatively like to the intrinsic concentration then the piezojunction effect at this current orientation is larger than at its longitudinal orientation. This fact is illustrated by Fig. 3 (curves (001) $)_{\text {trans }}$ and (001) long).

$$
\begin{aligned}
& \frac{n_{i}^{2}(\hat{u})}{n_{i}^{2}(0)}=\frac{1}{3}\left[\frac{m_{h}^{3 / 2} \exp \left(\delta E_{v}^{h} / k T\right)+m_{l}^{3 / 2} \exp \left(\delta E_{v}^{l} / k T\right)+m_{s-o}^{3 / 2} \exp \left(\left(\delta E_{v}^{s-o}-\Delta_{s-o}\right) / k T\right)}{m_{h}^{3 / 2}+m_{l}^{3 / 2}+m_{s-o}^{3 / 2} \exp \left(-\Delta_{s-o} / k T\right)}\right] \\
& \sum_{m=1}^{3} \exp \left(-\delta E_{c}^{(m)} / k T\right)
\end{aligned}
$$

\footnotetext{
${ }^{1}$ Using by occasion let us notice that in all figures of papers $[4,5]$ horizontal axes have to be reversed.
} 


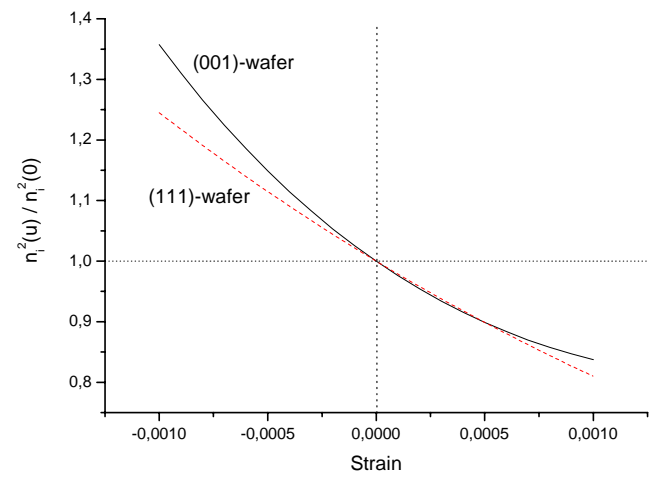

Fig. 1. Relative changes of squared intrinsic carrier concentration with bisotropic strain in silicon wafers of two orientations.

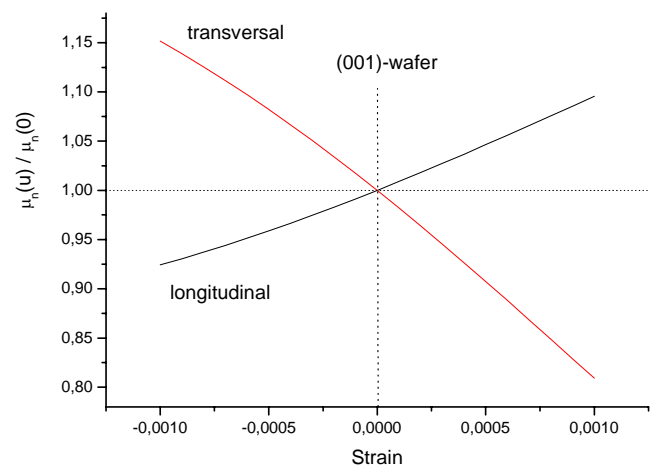

Fig. 2. Relative changes of the electron mobility with bisotropic strain in (001)-Si wafer at the current in plane of the wafer (longitudinal) and perpendicular to it (transversal).

\section{The case of (111)-oriented silicon wafer}

In this case, it is necessary to transform the initial bisotropic strain into crystallographic coordinate system by means of the transformation matrix [14]

$$
\left(\begin{array}{ccc}
1 / \sqrt{2} & \cos \theta / \sqrt{2} & \sin \theta / \sqrt{2} \\
-1 / \sqrt{2} & \cos \theta / \sqrt{2} & \sin \theta / \sqrt{2} \\
0 & -\sin \theta & \cos \theta
\end{array}\right)
$$

at $\sin \theta=\sqrt{2 / 3}, \cos \theta=\sqrt{1 / 3}$. As a result, for the same in magnitude initial (i.e. in the wafer plane) bisotropic stress (equal to $u_{\|} /\left(S_{11}+S_{12}\right)$ )

$u_{x x}=u_{y y}=u_{z z}=\frac{1}{3} \frac{S_{11}+2 S_{12}}{S_{11}+S_{12}} u_{\|}$,

$u_{x y}=u_{y z}=u_{z x}=\frac{1}{3} \frac{S_{44}}{S_{11}+S_{12}} u_{\|}$.

Under action of the strain (10) all three pairs of electron valleys take equal shifts

$$
\begin{aligned}
& \delta E_{c}^{(100)}=\delta E_{c}^{(010)}=\delta E_{c}^{(001)}= \\
& =2\left(\Xi_{d}+\frac{1}{3} \Xi_{u}\right) \frac{S_{11}+2 S_{12}}{S_{11}+S_{12}} u_{\|},
\end{aligned}
$$

and shifts in the energy of the valence subbands have the form (4) with different value of the parameter $\varepsilon=\frac{d}{2 \sqrt{3}} \frac{S_{44}}{S_{11}+S_{12}} u_{\|}$where $d$ is the $3^{\text {rd }}$ deformation potential for the valence band. Variation in the electron mobility does not occur at this wafer orientation: $\frac{\mu_{n}^{\text {trans }}(\hat{u})}{\mu_{n}(0)}=\frac{\mu_{n}^{\text {long }}(\hat{u})}{\mu_{n}(0)}=1$;

therefore the whole piezojunction effect is determined by variation of the squared intrinsic carrier concentration. The latter is represented by the curve "(111)wafer” in Fig. 1.

As is followed from comparison of two curves in Fig. 1 (as well as from Fig. 3) $p$ - $n$ junction on (111)oriented silicon wafer is less subjected to the influence of bisotropic compressions in comparison with (001)oriented one. As for bisotropic tensions, then (as followed from Fig. 3) their influence in the case of (001)-wafer can be both less and more than in the case of (111)-wafer depending on the current direction.

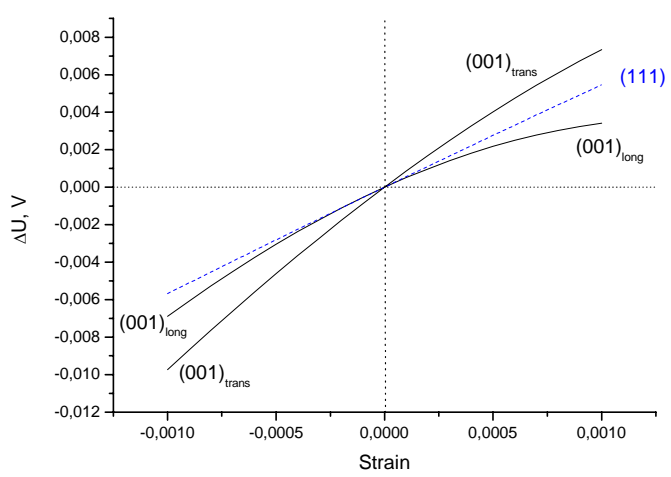

Fig. 3. Changes in the voltage drop across the diode versus bisotropic strain in (001)-Si wafer at the current in plane of the wafer (001) long and perpendicular to it (001) trans as well as in (111)-Si wafer (dashed line).

\section{The case of strong deformation of (001)-oriented silicon wafer}

In recent years, the great attention is attracted to use (for enhancement of electron and hole mobility) of biaxial strains induced in Si layer deposited onto SiGe buffer layer. The strains can achieve very large values. In particular, biaxial tensile strain of $0.8 \%$ has been measured in [15]. In the paper [16], the strain amounted to $1 \%$, in [17] - $1.2 \%$. Magnitude of the biaxial strain as large as $1.7 \%$ has been quoted in the review [18]. The statement about a method "that allows strain levels of up to and over $2 \%$ to be achieved either uniaxially or biaxially” was made in paper [19]. 

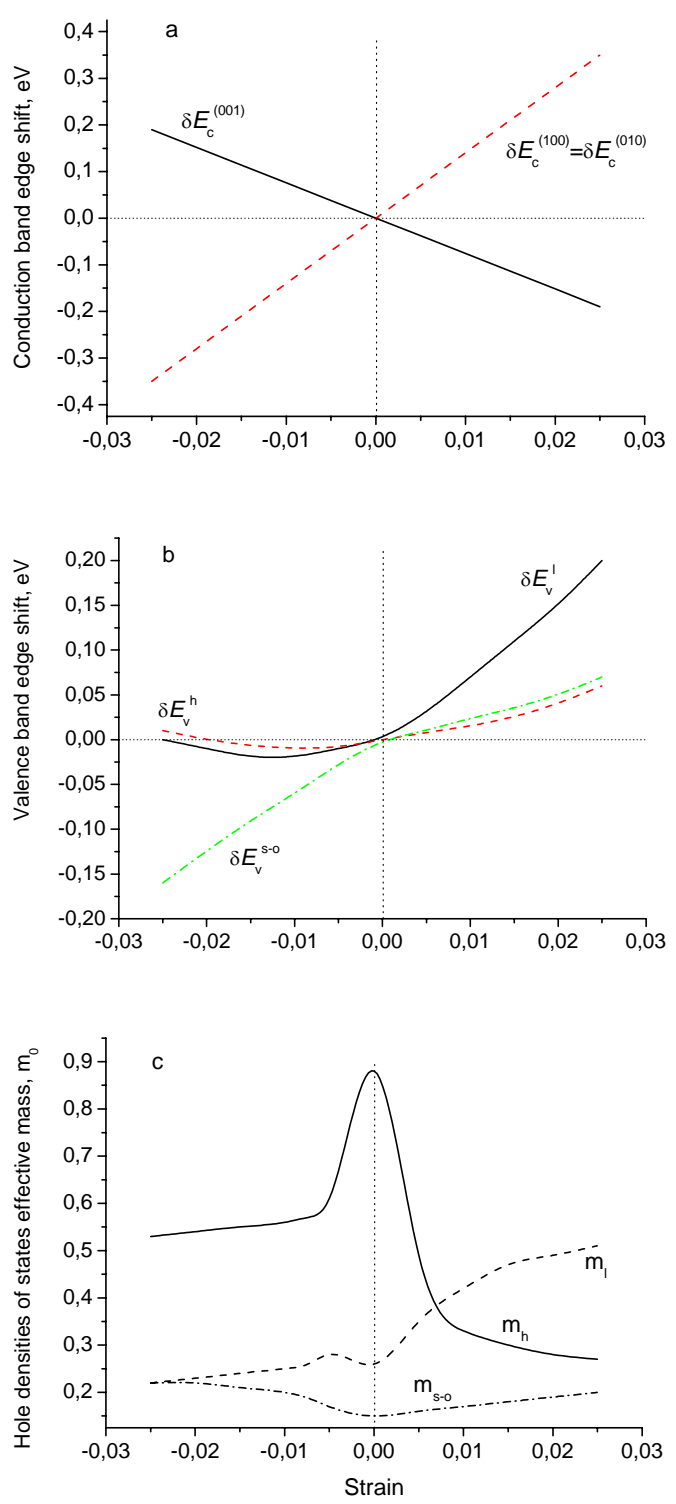

Fig. 4. Dependences of the conduction band edge shifts (a), the valence band edge shifts (b), and the hole densities-of-states effective masses (c) on large bisotropic strains in (001)-Si (from Ref. [20]).

Energy band structure and effective masses for strongly strained (001)-Si have been calculated in the paper [20]. In Fig. 4, these results have been reproduced, for convenience, in our designations. And shown in Fig. 5 is variation of the squared intrinsic carrier concentration calculated in accordance with these data for strain values achieving $\pm 2.5 \%$. As is seen from Fig. 5 intrinsic carrier concentration in strained silicon can increase under these conditions by several orders of magnitude (irrespective of deformation sign). So, lightly doped material can be transformed into material with intrinsic conductivity. As for $p$ - $n$ junction diode based on this material, the injection level of the emitter (at the given doping level) can increase, under these conditions, by five orders of magnitude.

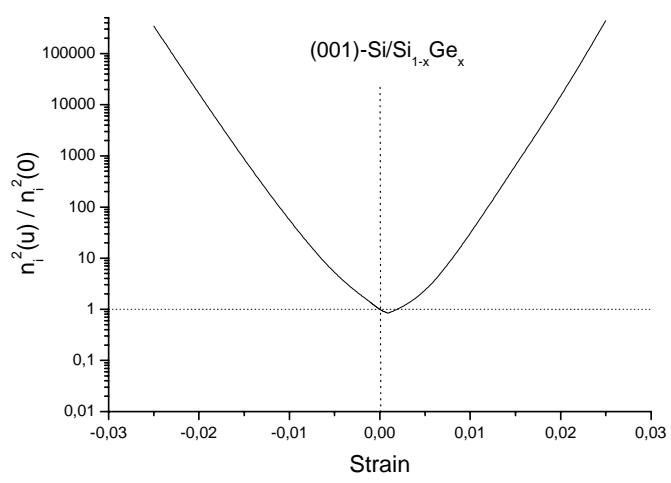

Fig. 5. Relative change of squared intrinsic carrier concentration with bisotropic strain in (001)-Si at large bisotropic strains (as based on the results from Fig. 4).

\section{Conclusion}

So, as for effect of the bisotropic strains on indications of diode temperature sensors, the diodes on the surface of (111)-Si are less subjected to their influence (in the case of bisotropic compression) in comparison with (001)-oriented Si. Furthermore, though at small strains, the band gap of silicon that determines the intrinsic carrier concentration narrows at bisotropic compressions and broadens at bisotropic tensions, at large bisotropic strains of any sign, narrowing the band gap and significant growth of the intrinsic carrier concentration take place.

\section{References}

1. J.J. Wortman, J.R. Hauser, and R.M. Burger, Effect of mechanical stress on $p$-n junction device characteristics // J. Appl. Phys. 35 (7), p. 21222131 (1964).

2. Y. Kanda, Effect of stress on germanium and silicon $p$-n junction // Jpn. J. Appl. Phys. 6 (4), p. 475-486 (1967).

3. J.F. Creemer and P.J. French, The piezojunction effect in bipolar transistors at moderate stress level: a theoretical and experimental study // Sensors and Actuators A 82 (1-3), p. 181-185 (2000).

4. V.L. Borblik, Yu.M. Shwarts, and E.F. Venger, Effect of mechanical stress on operation of diode temperature sensors // Semiconductor Physics, Quantum Electronics \& Optoelectronics 5 (3), p. 322-327 (2002).

5. V.L. Borblik, Yu.M. Shwarts, and E.F. Venger, About manifestation of the piezojunction effect in diode temperature sensors // Semiconductor Physics, Quantum Electronics \& Optoelectronics 6 (1), p. 97-101 (2003).

6. J.F. Creemer and P.J. French, The saturation current of silicon bipolar transistors at moderate stress levels and its relation to the energy-band structure // J. Appl. Phys. 96 (8), p. 4530-4538 (2004). 
7. J.-S. Lim, S.E. Thompson, and J.G. Fossum, Comparison of threshold-voltage shifts for uniaxial and biaxial tensile-stressed n-MOSFETs // IEEE Electron Device Lett. 25 (11), p. 731-733 (2004).

8. M.P.F. de Godoy, M.K.K. Nakaema, F. Iikawa, W. Carvalho, Jr., E. Ribeiro, and A.L. Gobbi, Biaxial stress ring applications to magneto-optical studies of semiconductor films // Rev. Sci. Instr. 75 (6), p. 1947-1951 (2004).

9. S. Maikap, C.-Y. Yu, S.-R. Jan, M.H. Lee, C.W. Liu, Mechanically strained strained-Si nMOSFETs // IEEE Electron Device Lett. 25 (1), p. 40-42 (2004).

10. B.M. Haugerud, M.B. Nayeema, R. Krithivasan, Y. Lu, C. Zhu, J.D. Cressler, R.E. Belford, and A.J. Joseph, The effects of mechanical planar biaxial strain in Si/SiGe HBT BiCMOS technology // Solid-State Electronics 49 (6), p. 986-990 (2005).

11. F.J. Beisswanger and H. Jorke, Electron transport in bipolar transistors with biaxially strained base layers // IEEE Trans. Electron Devices 43 (1), p. 62-69 (1996).

12. E. Anastassakis, Strained superlattices and heterostructures: Elastic consideration // J. Appl. Phys. 68 (9), p. $4561-4568$ (1990).

13. H. Hasegawa, Theory of cyclotron resonance in strained silicon crystals // Phys. Rev. 129 (3), p. 1029-1040 (1963).

14. J.C. Hensel and G. Feher, Cyclotron resonance experiments in uniaxially stressed silicon: valence band inverse mass parameters and deformation potentials // Phys. Rev. 129 (3), p. 1041-1062 (1963).
15. K. Arimoto, D. Furukawa, J. Yamanaka, K. Nakagawa, K. Sawano, S. Koh, Y. Shiraki, and $\mathrm{N}$. Usami, Changes in elastic deformation of strained Si by microfabrication // Mater. Sci. Semicond. Process. 8 (1-3), p.181-185 (2005).

16. T.K.S. Wong, Y. Gong, P. Yang, and C.M. Ng, Characterization of biaxial stressed silicon by spectroscopic ellipsometry and synchrotron x-ray scattering // Semicond. Sci. Technol. 22 (11), p. 1232-1239 (2007).

17. N. Hirashita, Y. Moriyama, N. Sugiyama, E. Toyoda, and S. Takagi, Strain relaxation in strained-Si layers on SiGe-on-insulator substrates // Semicond. Sci. Technol. 22 (1), p. S21-S25 (2007).

18. M.L. Lee, E.A. Fitzgerald, M.T. Bulsara, M.T. Currie, and A. Lochtefeld, Strained Si, SiGe, and Ge channels for high-mobility metal-oxidesemiconductor field-effect transistors // J. Appl. Phys. 97 (1), 011101/1-27 (2005).

19. B.M. Haugerud, L.A. Bosworth, and R.E. Belford, Elevated-temperature electrical characteristics of mechanically strained Si devices // J. Appl. Phys. 95 (5), p. 2792-2796 (2004).

20. M.V. Fischetti and S.E. Laux, Band structure, deformation potentials and carrier mobility in strained Si, Ge, and SiGe alloys // J. Appl. Phys. 80 (4), p. 2234-2252 (1996). 